\title{
Supporting Information for Enhancing water sampling in free energy calculations with grand canonical Monte Carlo
}

\author{
Gregory A. Ross*, Ellery Russell, Yuqing Deng, Chao Lu, Edward D. Harder, \\ Robert Abel, Lingle Wang* \\ Schrödinger, Inc., 120 West 45th Street, New York, New York 10036, United States \\ E-mail: gregory.ross@schrodinger.com; lingle.wang@schrodinger.com
}

\section{The equivalence of density fluctuations in NPT and $\mu \mathrm{VT}$ ensem- bles}

Equation 1 in the main text states that density fluctuations in the NPT and $\mu \mathrm{VT}$ ensembles are equivalent for pure systems of the same size. To derive this result, we begin by stating relations that can be found in most statistical mechanics textbooks.

In the NPT ensemble, the variance of the volume is given by

$$
\operatorname{Var}(V)_{N P T}=-k_{B} T \frac{\partial\langle V\rangle_{N P T}}{\partial P}
$$

where angular brackets denote ensemble averages. Similarly, the variance of the number of particles in the $\mu \mathrm{VT}$ ensemble is given by

$$
\operatorname{Var}(N)_{\mu V T}=k_{B} T \frac{\partial\langle N\rangle_{\mu V T}}{\partial \mu}
$$

The isothermal compressibility, $\kappa_{T}$, relates the response of a system to changes in pressure or chemical potential at a fixed temperature. In the NPT ensemble, it is defined as

$$
\kappa_{T} \equiv-\frac{1}{\langle V\rangle_{N P T}} \frac{\partial\langle V\rangle_{N P T}}{\partial P} .
$$

and in the $\mu \mathrm{VT}$ ensemble, it can be shown that

$$
\kappa_{T}=\frac{V}{\langle N\rangle_{\mu V T}^{2}} \frac{\partial\langle N\rangle_{\mu V T}}{\partial \mu}
$$

Putting equations 1-4 together we have

$$
\frac{1}{\langle V\rangle_{N P T}} \operatorname{Var}(V)_{N P T}=\frac{V}{\langle N\rangle_{\mu V T}^{2}} \operatorname{Var}(N)_{\mu V T}
$$

We therefore have a correspondence between the fluctuation of the volume in the NPT ensemble and the fluctuation of the number particles in the $\mu \mathrm{VT}$ ensemble. The last part of this derivation reinterprets the above fluctuations as changes in density, defined as $\rho \equiv N / V$.

To proceed, we will require the following approximation

$$
\operatorname{Var}\left(\frac{1}{V}\right) \approx \frac{\operatorname{Var}(V)}{\langle V\rangle^{4}},
$$

which is valid in the large $V$ limit and can be derived using a Taylor expansion. For a N, the variance of the 
density is given by

$$
\begin{aligned}
\operatorname{Var}(\rho)_{N P T} & =\left\langle\rho^{2}\right\rangle_{N P T}-\langle\rho\rangle_{N P T}^{2} \\
& =N^{2}\left\langle\frac{1}{V^{2}}\right\rangle_{N P T}-N^{2}\left\langle\frac{1}{V}\right\rangle_{N P T}^{2} \\
& \approx \frac{N^{2}}{\langle V\rangle_{N P T}^{4}} \operatorname{Var}(V)_{N P T} .
\end{aligned}
$$

In the $\mu \mathrm{VT}$ ensemble, it is straightforward to show that

$$
\operatorname{Var}(\rho)_{\mu V T}=\frac{1}{V^{2}} \operatorname{Var}(N)_{\mu V T}
$$

Putting equations 7 and 8 into equation 5 yields the desired relation between the density fluctuations in both ensembles:

$$
\frac{\langle V\rangle_{N P T}}{\langle\rho\rangle_{\mathrm{NPT}}^{2}} \operatorname{Var}(\rho)_{N P T} \approx \frac{V_{\mu \mathrm{VT}}}{\langle\rho\rangle_{\mu \mathrm{VT}}^{2}} \operatorname{Var}(\rho)_{\mu \mathrm{VT}},
$$

The approximate relationship becomes exact in the thermodynamic limit, i.e. when $N \rightarrow \infty$ and $V \rightarrow \infty$ for a finite $\rho$.

\section{Free energy calculations in $\mu \mathrm{VT}$}

\subsection{Constructing free energy estimators in the $\mu \mathrm{VT}$ ensemble}

This section illustrates that when perturbations are limited to the potential energy, free energy estimators, such as exponential averaging and Bennett's accepatance ratio method (BAR), are the same in $\mu$ VT as the NVT and NPT ensembles. No additional terms or corrections are required in the $\mu \mathrm{VT}$ ensemble to account for the variable number of particles.

First, we note that the partition function in the $\mu \mathrm{VT}$ ensemble is given by

$$
Z(\mu, V, T)=\sum_{N=0}^{\infty} \frac{1}{N !} \int e^{\beta\left[\mu N-E\left(x^{N}, N\right)\right]} d x^{N}
$$

where $x^{N}$ is the configuration for the $N$ particles. We next introduce a control parameter, $\lambda$, that can alter the potential energy, now denoted $E\left(\lambda, x^{N}, N\right)$. The goal is to calculate the relative free energy between two endpoints $\lambda_{0}$ and $\lambda_{1}$, which will require the evaluation of the ratio $Z\left(\lambda_{0}, \mu, V, T\right) / Z\left(\lambda_{1}, \mu, V, T\right)$.

Using the exponential averaging equation, we now show that - because the control parameters only affect the potential energy - we can construct an estimator for the ratio of partition functions in the $\mu$ VT ensemble in exactly the same way as in the NPT and NVT ensemble. The equivalent procedure carries over to other common free energy estimators, such as BAR and thermodynamic integration.

$$
\begin{aligned}
\frac{Z\left(\lambda_{0}, \mu, V, T\right)}{Z\left(\lambda_{1}, \mu, V, T\right)} & =\frac{1}{Z\left(\lambda_{1}, \mu, V, T\right)} \sum_{N=0}^{\infty} \frac{e^{\beta N}}{N !} \int e^{-\beta E\left(\lambda_{0}, x^{N}, N\right)} d x^{N} \\
& =\frac{1}{Z\left(\lambda_{1}, \mu, V, T\right)} \sum_{N=0}^{\infty} \frac{e^{\beta N}}{N !} \int e^{-\beta E\left(\lambda_{0}, x^{N}, N\right)} e^{\beta E\left(\lambda_{1}, x^{N}, N\right)} e^{-\beta E\left(\lambda_{1}, x^{N}, N\right)} d x^{N} \\
& =\frac{1}{Z\left(\lambda_{1}, \mu, V, T\right)} \sum_{N=0}^{\infty} \frac{e^{\beta N}}{N !} \int e^{-\beta \Delta E\left(x^{N}, N\right)} e^{-\beta E\left(\lambda_{1}, x^{N}, N\right)} d x^{N} \\
& =\left\langle e^{-\beta \Delta E\left(x^{N}, N\right)}\right\rangle_{\lambda_{1} \mu V T},
\end{aligned}
$$

where $\Delta E\left(x^{N}, N\right) \equiv E\left(\lambda_{0}, x^{N}, N\right)-E\left(\lambda_{1}, x^{N}, N\right)$. The main point is that as the perturbation only affects the potential energy no special considerations are required for the fluctuating particle number. The grand canonical ensemble average, denoted as \langle\rangle$_{\lambda_{1} \mu V T}$, can be estimated in the usual way, i.e. by running a simulation in the $\mu \mathrm{VT}$ ensemble and calculating the mean. 


\subsection{Equivalence of free energy estimators in NPT and $\mu \mathrm{VT}$ ensembles}

This section explores the difference between free energies calculated in the grand canonical ensemble $(\mu V T)$, the isobaric-isothermal $(N P T)$, and canonical ensemble $(N V T)$. The strategy uses Taylor series expansions of the different estimating equations for free energies. Central to this approach is the fact that expectation values in the $\mu \mathrm{VT}$ and NPT ensembles can be expressed as expectations over the NVT ensemble. The procedure is demonstrated on thermodynamics integration and can be similarly applied to any other free energy estimation technique such as exponential averaging and BAR.

Let $A=A\left(x^{N}, N, V\right)$ be some function of the state of the system. One can show that the expectation value of $A$ in the $\mu \mathrm{VT}$ ensemble can be expressed as

$$
\langle A\rangle_{\mu V T}=\sum_{N=0}^{\infty} p(N \mid \mu, V, T)\langle A\rangle_{N V T},
$$

where $p(N \mid \mu, V, T)$ is the probability mass function for the number of molecules in the grand canonical ensemble and \langle\rangle$_{N V T}$ denotes an expectation value in the NVT ensemble. For the isobaric-isothermal ensemble, a similar relationship holds:

$$
\langle A\rangle_{N P T}=\int d V p(V \mid N, P, T)\langle A\rangle_{N V T}
$$

where $p(V \mid N, P, T)$ is the probability density function for observing a particular volume given a particular thermodynamic state.

These relationships allow us to use the following second order Taylor expansion for the grand canonical ensemble

$$
\begin{aligned}
\langle A\rangle_{\mu V T} & \approx\langle A\rangle_{\langle N\rangle_{\mu V T}}+\left.\frac{1}{2} \operatorname{Var}_{\mu V T}(N) \frac{\partial^{2}\langle A\rangle_{N V T}}{\partial N^{2}}\right|_{\langle N\rangle_{\mu V T}} \\
& =\langle A\rangle_{\langle\rho\rangle_{\mu V T}}+\left.\frac{1}{2} \operatorname{Var}_{\mu V T}(N) \frac{\partial^{2}\langle A\rangle_{N V T}}{\partial N^{2}}\right|_{\langle N\rangle_{\mu V T}}
\end{aligned}
$$

and isobaric-isothermal ensemble

$$
\langle A\rangle_{N P T} \approx\langle A\rangle_{\langle\rho\rangle_{N P T}}+\left.\frac{1}{2} \operatorname{Var}_{N P T}(V) \frac{\partial^{2}\langle A\rangle_{N P T}}{\partial V^{2}}\right|_{\langle V\rangle_{N P T}},
$$

where $\operatorname{Var}_{\mu V T}(N)$ denotes the variance of $N$ in the grand canonical ensemble, and similarly for $\operatorname{Var}_{N P T}(V)$.

Free energy estimators, such as exponential averaging, thermodynamic integration (TI), and BAR, are ensemble averages of particular functions. The Taylor series expansions above show that we can treat estimators in the $\mu \mathrm{VT}$ and NPT ensembles as perturbations of the canonical ensemble. The chemical potential of water is calibrated so that water densities are equal between the $\mu \mathrm{VT}$ and NPT ensembles. As we are concerned with system composed overwhelmingly of water, the first order terms (i.e. $\langle A\rangle_{\rho_{\mu V T}}$ and $\langle A\rangle_{\langle\rho\rangle_{N P T}}$ ) are approximately equal. The following uses TI as an example to show that these second-order terms are small and approximately equal, such that free energies calculated in the $\mu \mathrm{VT}$ ensemble are approximately equal to free energies calculated in the NPT ensemble in at least the first and second order terms of the above expansion.

As before, let $E=E\left(\lambda, x^{N}, N\right)$ denote the total energy of the system and $\lambda$ denote the alchemical coordinate. The system under consideration consists of a solute immersed in water. Mimicking small molecule free energy calculations, only the solute is directly perturbed by $\lambda$; the solvent only depends on $\lambda$ by interacting with the solute. In the $\mu \mathrm{VT}$, the free energy difference, $\Delta F \equiv F\left(\lambda_{1}, \mu, V, T\right)-F\left(\lambda_{0}, \mu, V, T\right)$, between the alchemical states $\lambda=0$ and $\lambda=1$ is given by

$$
\Delta F(\mu, V)=\int_{0}^{1}\left\langle\frac{\partial E}{\partial \lambda}\right\rangle_{\mu \lambda V} d \lambda
$$

where the dependence on the temperature has been suppressed to simplify the notation. To relate this grand canonical free energy difference to a Helmholtz free energy difference, we can expand the integrand about the mean particle number using equation 13 to get

$$
\left\langle\frac{\partial E}{\partial \lambda}\right\rangle_{\mu V \lambda} \approx\left\langle\frac{\partial E}{\partial \lambda}\right\rangle_{\langle\rho\rangle_{\mu V \lambda}}+\left.\frac{1}{2} \operatorname{Var}_{\lambda}(N) \frac{\partial^{2}}{\partial N^{2}}\left\langle\frac{\partial E}{\partial \lambda}\right\rangle_{N V \lambda}\right|_{\langle N\rangle_{\mu V \lambda}} .
$$


The benefit of this expansion is that we can compare grand canonical expectation values with canonical expectation values when the canonical ensemble is composed of the mean number of particles in the grand canonical ensemble. The second-order term in the above can be simplified in the following way:

$$
\begin{aligned}
\left.\frac{\partial^{2}}{\partial N^{2}}\left\langle\frac{\partial E}{\partial \lambda}\right\rangle_{N, V, \lambda}\right|_{\langle N\rangle_{\mu V \lambda}} & =\left.\frac{\partial^{2}}{\partial N^{2}} \frac{\partial F}{\partial \lambda}\right|_{\langle N\rangle_{\mu V \lambda}} \\
& =\left.\frac{\partial}{\partial \lambda} \frac{\partial}{\partial N} \frac{\partial F}{\partial N}\right|_{\langle N\rangle_{\mu V \lambda}} \\
& =\left.\left.\frac{\partial}{\partial \lambda} \frac{\partial \mu}{\partial N}\right|_{\langle N\rangle_{\mu V \lambda}}\right|^{-1} \\
& =\frac{\partial}{\partial \lambda}\left(\frac{\partial\langle N\rangle_{\mu V T}}{\partial \mu}\right)^{\frac{1}{\partial \lambda}} \frac{1}{\operatorname{Var}(N)_{\mu V \lambda}} \\
& =k_{B} T \frac{\partial}{\partial)^{\prime}}
\end{aligned}
$$

where $\mu \equiv \partial F / \partial N$ is the chemical potential of the system. The last line follows from equation 2 . So, for the $\mu \mathrm{VT}$ ensemble, we have

$$
\begin{aligned}
\left\langle\frac{\partial E}{\partial \lambda}\right\rangle_{\mu V \lambda} & \approx\left\langle\frac{\partial E}{\partial \lambda}\right\rangle_{\langle\rho\rangle_{\mu V \lambda}}+\frac{1}{2} k_{B} T \operatorname{Var}(N)_{\mu V \lambda} \frac{\partial}{\partial \lambda} \frac{1}{\operatorname{Var}(N)_{\mu V \lambda}} \\
& =\left\langle\frac{\partial E}{\partial \lambda}\right\rangle_{\langle\rho\rangle_{\mu V \lambda}}+\frac{1}{2} k_{B} T \operatorname{Var}(\rho)_{\mu V \lambda} \frac{\partial}{\partial \lambda} \frac{1}{\operatorname{Var}(\rho)_{\mu V \lambda}}
\end{aligned}
$$

Similarly, one can show for the NPT ensemble that

$$
\left\langle\frac{\partial E}{\partial \lambda}\right\rangle_{N P \lambda} \approx\left\langle\frac{\partial E}{\partial \lambda}\right\rangle_{\langle\rho\rangle_{N P \lambda}}+\frac{1}{2} k_{B} T \operatorname{Var}(\rho)_{N P \lambda} \frac{\partial}{\partial \lambda} \frac{1}{\operatorname{Var}(\rho)_{N P \lambda}} .
$$

Thus, we find that the mean gradient of the energy has a similar form between the $\mu \mathrm{VT}$ and NPT ensembles. As described above, if the chemical potential in the grand canonical ensemble has been calibrated to match the water density in the NPT ensemble, then the first order terms should be equal, i.e.

$$
\left\langle\frac{\partial E}{\partial \lambda}\right\rangle_{\langle\rho\rangle_{N P \lambda}}=\left\langle\frac{\partial E}{\partial \lambda}\right\rangle_{\langle\rho\rangle_{\mu V \lambda}}
$$

The second order terms depends on the variance of the density between the ensembles, which Section 1 (equation 1 of the main text) shows to be equal between the $\mu \mathrm{VT}$ and NPT ensembles for large systems of equal size. When the solvent only depends on $\lambda$ via the interaction with the solute, we expect the response of the solvent density to be approximately equal between the $\mu \mathrm{VT}$ and NPT ensembles, i.e.

$$
\frac{\partial}{\partial \lambda} \frac{1}{\operatorname{Var}(\rho)_{\mu V \lambda}} \approx \frac{\partial}{\partial \lambda} \frac{1}{\operatorname{Var}(\rho)_{N P \lambda}} .
$$

We have therefore shown that

$$
\left\langle\frac{\partial E}{\partial \lambda}\right\rangle_{\mu V \lambda} \approx\left\langle\frac{\partial E}{\partial \lambda}\right\rangle_{N P \lambda}
$$

As the above are integrated to get the free energy changes in the $\mu \mathrm{VT}$ and NPT ensembles, we have thus shown that $\Delta F(\mu, V) \approx \Delta F(N, P)$.

\section{The thermodynamics of water displacement}

Consider two hypothetical ligands, $a$ and $b$, that can bind to a receptor. Ligand $a$ is larger than ligand $b$ such that ligand $b$ can accommodate a single buried water molecule. This water molecule resides in the bulk solvent when ligand $a$ is bound. Figure 1 in the main text shows schematic representations of these systems. We are 
interested in the relative binding free energy between these two ligands, and, in particular, the contribution of the buried water molecule. The relative binding free energy to transmute ligand $a$ to $b$ is given by

$$
\Delta \Delta G_{a \rightarrow b}=-k_{B} T \ln \left(\frac{Z_{\mathrm{solv}}^{a} Z_{\mathrm{rec}}^{b}}{Z_{\mathrm{rec}}^{a} Z_{\mathrm{solv}}^{b}}\right),
$$

where $Z_{\text {solv }}$ denotes the partition functions for a ligand when it is unbound and in solvent, $Z_{\text {rec }}$ denotes the partition function for a ligand when it is bound to the receptor, and $k_{B} T$ denotes thermal energy.

We can understand the influence of the water molecule on the affinity of ligand $b$ by explicitly writing the partition function for ligand $b$ when it is bound to the receptor. Let the system contain $N$ identical water molecules with coordinates $x^{N} \equiv\left(x_{1}, x_{2}, \ldots, x_{N}\right)$ and total potential energy $E\left(x^{N}\right)$. The coordinates of the receptor and ligand will be ignored to simplify the notation. The partition function of the bound state of ligand $b$ is then given by

$$
Z_{\mathrm{rec}}^{b}=\frac{1}{N !} \int e^{-\beta E\left(x^{N}\right)} d x^{N},
$$

where the integral is over all degrees of freedom within the system volume and $\beta=1 / k_{B} T$. As suggested by Figure 1 in the main text, let us suppose that water can be in two states: bound to the protein in the buried pocket or unbound in the bulk solvent. This two state description is valid for more complex systems when the volume of the solvent is much larger than the free volume within the receptor. Indicator functions can be introduced into $Z_{\mathrm{rec}}^{b}$ to distinguish between the two allowed states of the water: $I_{\text {rec }}\left(x_{i}\right)=1$ if water $i$ is bound to the receptor and zero otherwise, and $I_{\text {solv }}\left(x_{i}\right)=1$ if water $i$ is in the solvent and zero otherwise. By construction we have $I_{\text {solv }}\left(x_{i}\right)+I_{\text {rec }}\left(x_{i}\right)=1 \forall x_{i}$ so we can rewrite $Z_{\text {rec }}^{b}$ as

$$
Z_{\mathrm{rec}}^{b}=\frac{1}{N !} \int e^{-\beta E\left(x^{N}\right)} \prod_{i=1}^{N}\left(I_{\mathrm{solv}}\left(x_{i}\right)+I_{\mathrm{rec}}\left(x_{i}\right)\right) d x^{N} .
$$

The product of indicator functions can be expanded and all terms where there are $n=1,2, . ., N$ water molecules in the receptor and in solvent can be collected to give

$$
Z_{\text {rec }}^{b}=\sum_{n=0}^{N} \frac{1}{n !(N-n) !} \int_{\substack{\text { N bound } \\ \text {-n unbound }}} e^{-\beta E\left(x^{N}\right)} d x^{N},
$$

where the domains of the integrals refer to the number of water molecules that are bound to the receptor $(n)$ and unbound in the solvent $(N-n)$.

\subsection{Displacing a maximum of one buried water molecule}

When the water cavity can only contain one or zero water molecules, such as in Figure 1, the partition function for when ligand $b$ is bound reduces to

$$
\begin{aligned}
Z_{\text {rec }}^{b} & =\frac{1}{N !} \int_{\substack{0 \\
\text { bound unbound }}} e^{-\beta E\left(x^{N}\right)} d x^{N}+\frac{1}{(N-1) !} \int_{\substack{1 \text { bound } \\
\text { b-1 unbound }}} e^{-\beta E\left(x^{N}\right)} d x^{N} \\
& =\frac{1}{N !} \int_{\substack{0 \\
\text { bound unbound }}} e^{-\beta E\left(x^{N}\right)} d x^{N}\left(1+N \frac{\int_{\substack{1 \\
\text { bound }-1 \text { unbound }}} e^{-\beta E\left(x^{N}\right)} d x^{N}}{\int_{\substack{0 \\
\text { bound unbound }}} e^{-\beta E\left(x^{N}\right)} d x^{N}}\right) \\
& =Z_{\text {rec }}^{b, 0 \text { bound }}(1+R),
\end{aligned}
$$

which can be interpreted as the partition function for when ligand $b$ is bound to the receptor when no waters are bound, denoted as $Z_{\text {rec }}^{b, 0}$ bound, multiplied by a solvent correction factor, $(1+R)$. Insight can be gained on the ratio of integrals in the solvent correction factor by introducing the potential $E\left(x^{N-1}\right)$, which represents the potential energy of the system when one of the water molecules has been decoupled from the system, i.e. is in an ideal gas state. The internal energy of the decoupled water molecule will be ignored to help simplify the 
notation.

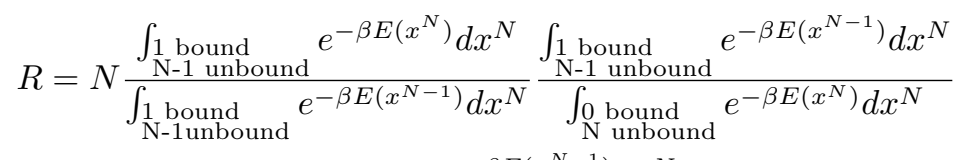

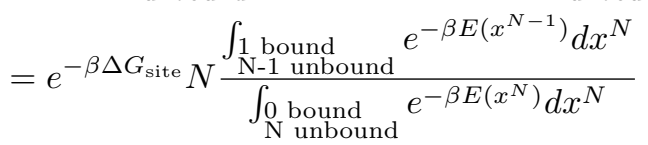

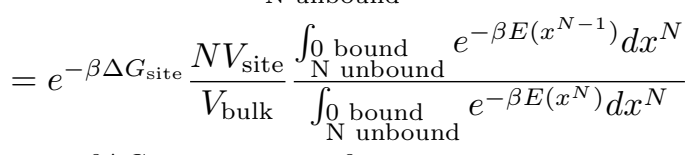

$$
\begin{aligned}
& =e^{-\beta \Delta G_{\text {site }}} \rho_{\text {bulk }} V_{\text {site }} e^{\beta \mu_{\text {ex }}} \\
& =e^{\beta\left[\mu_{\text {ex }}-\Delta G_{\text {site }}+k_{B} T \ln \left(\rho_{\text {bulk }} V_{\text {site }}\right)\right]} \\
& =e^{-\beta \Delta G_{\text {water }}^{\text {bind }}}
\end{aligned}
$$

From the second line, $\Delta G_{\text {site }}$ is used to represent the free energy to couple a water molecule to the hydration site in the receptor. For $\Delta G_{\text {site }}$, a water molecule is restricted to the hydration site in both the coupled and decoupled state. In the third line, the integral in the numerator is converted to represent the state where a water molecule is instead restricted to be in bulk water and not in the bound state. This conversion requires the ratio of the volume of the hydration site, $V_{\text {site }}$, and the volume of bulk water $V_{\text {bulk }}$. In the fourth line, the free energy to couple a water molecule from ideal gas to bulk water (equivalent to the excess chemical potential), $\mu_{\mathrm{ex}}$, is introduced, as is the density of bulk water $\rho_{\text {bulk }}$. The free energies $\Delta G_{\text {site }}$ and $\mu_{\text {ex }}$ can be calculated in molecular simulations via alchemical decoupling techniques. The term $k_{B} T \ln \left(\rho_{\text {bulk }} V_{\text {site }}\right)$ represents the concentration correction to move a water molecule from the receptor to bulk water. In the final line, all the terms in the exponent are collected and re-interpreted as the standard free energy to bind a single water molecule from bulk to the hydration site, denoted $\Delta G_{\mathrm{water}}^{\text {bind }}$. Therefore, for the partition function of the bound state of ligand $b$, we have

$$
Z_{\mathrm{rec}}^{b}=Z_{\mathrm{rec}}^{b, 0 \text { bound }}\left(1+e^{-\beta \Delta G_{\text {water }}^{\text {bind }}}\right) .
$$

Returning to the relative free energy to transform ligand $a$ to ligand $b$ in equation 23 , we now have

$$
\begin{aligned}
\Delta \Delta G_{a \rightarrow b} & =-k_{B} T \ln \left(\frac{Z_{\text {solv }}^{a} Z_{\text {rec }}^{b, 0 \text { bound }}}{Z_{\text {rec }}^{a} Z_{\text {solv }}^{b}}\right)-k_{B} T \ln \left(1+e^{-\beta \Delta G_{\text {water }}^{\text {bind }}}\right) \\
& =\Delta \Delta G_{a \rightarrow b}^{\mathrm{dry}}+\Delta \Delta G_{\text {solv }},
\end{aligned}
$$

where $\Delta \Delta G_{a \rightarrow b}^{\mathrm{dry}}$ is the relative binding free energy when the hydration site is unoccupied, i.e. $d r y$, and $\Delta \Delta G_{\text {solv }}$ is the free energy to solvate the hydration site. It is important to stress that $\Delta \Delta G_{\text {solv }}$ is always less than or equal to zero. The more negative $\Delta G_{\text {water }}^{\text {bind }}$ is, the more favorably a hydration will contribute favorably to the affinity. For a new ligand to displace a water molecule and have a lower (i.e. more favorable) binding free energy, the new ligand must be able to compensate for loss of hydration, for instance, by increasing the interaction with the receptor.

One can also derive a form the $\Delta \Delta G_{\text {solv }}$ in another way that can also inform its interpretation. Considering again the ratio $R$ in equation 27: it is the integral of all the states where the hydration site is occupied over the integral of all the states where the site is unoccupied. If $p$ denotes the probability that the site is occupied and $1-p$ the probability that the site is unoccupied, we have $R=p /(1-p)$ so that

$$
\begin{aligned}
\Delta \Delta G_{\mathrm{solv}} & =-k T \ln \left(1+\frac{p}{1-p}\right) \\
& =k T \ln (1-p) .
\end{aligned}
$$

The solvation free energy of the site is zero if and only if $p=0$. Otherwise, any occupancy of the hydration site i.e. $0<p \leq 1$ will contribute favorably to the binding affinity of the smaller ligand - ligand $b$ in the example above. 


\subsection{Displacing an arbitrary number of water molecules}

When the binding site can accommodate more than one water molecule, all of which are displaced by one of the ligands, the procedure used to derive equations 27 to 30 can be shown to give

$$
\Delta \Delta G_{\text {solv }}=-k_{B} T \ln \left(1+\sum_{n=1}^{N} \frac{1}{n !} e^{-\beta \Delta G_{\text {water }}^{\text {bind }}(n)}\right)
$$

where $\Delta G_{\text {water }}^{\text {bind }}(n)$ is the standard state free energy to bind $n$ water molecules to the binding site.

The standard state binding free energies, $\Delta G_{\text {water }}^{\text {bind }}(n)$, can be calculated using the decouple decoupling technique. For these calculations, the water molecules would be typically decoupled sequentially whilst being restrained or constrained to particular hydration sites. Restricting each water molecule to a single hydration site explicitly treats the water as distinguishable, which breaks the permutation symmetry of water. Hence, this procedure would overestimate the standard state binding free energy by $k_{B} T \ln (n !)$, which would cancel exactly with the $n$ ! term in equation 32 . That is, if the standard state binding free energy that is calculated by double decoupling with restrained water sites is denoted $\Delta G_{\mathrm{DD}}^{\text {bind }}$, then

$$
\Delta \Delta G_{\mathrm{solv}}=-k_{B} T \ln \left(1+\sum_{n=1}^{N} e^{-\beta \Delta G_{\mathrm{DD}}^{\mathrm{bind}}(n)}\right) .
$$

Furthermore, because $-\Delta G_{\text {water }}^{\text {bind }}(n)$ is exponentiated in equation 32 , the sum over water molecules is dominated by the term with the minumum (i.e. most negative) $\Delta G_{\text {water }}^{\text {bind }}(n)$. Thus, to a good approximation, the total solvent correction that is calculated by double decoupling is given by

$$
\Delta \Delta G_{\mathrm{solv}} \approx-k_{B} T \ln \left(1+e^{-\beta \Delta G_{\mathrm{DD}}^{\mathrm{bind}}\left(n^{*}\right)}\right)
$$

where $n^{*}=\min _{n}\left\{\Delta G_{\text {water }}^{\text {bind }}(n)\right\}$. This relation will be used to compare ligand binding free energies calculated in the $\mu \mathrm{VT}$ and NPT ensembles, where the solvent contribution in the NPT is calculated using double decoupling.

\section{The calibration of the chemical potential}

Along with volume and temperature, the chemical potential is one of the thermodynamic control parameters of the grand canonical ensemble. For a single chemical species, the chemical potential is given by

$$
\mu=\mu_{\mathrm{ex}}-k_{B} T \ln (\rho),
$$

where $\mu_{\mathrm{ex}}$ is the excess chemical potential, $\rho$ is the density, and $k_{B}$ is Boltzmann's constant. The chemical potential is the free energy to add a molecule to a reservoir that is in chemical equilibrium with the system of interest. In the case of sampling water with GCMC, we are interested in systems that are solvated in bulk water at room temperature and atmospheric pressure, so that is the appropriate reservoir. Thus, for our purpose, $\rho$ corresponds to the density of bulk water and $\mu_{\mathrm{ex}}$ the hydration free energy of water. Both of which will be calculated at 1 bar and $300 \mathrm{~K}$. The chemical potential, and therefore $\rho$ and $\mu_{\mathrm{ex}}$, only need to be calculated once for each water model, treatment of long ranged interactions, and thermodynamic state of the reservoir.

We calibrated $\mu$ by separately calculating $\mu_{\mathrm{ex}}$ and $\rho$. The separation of the chemical potential into these two parameters is not necessary for calibration but is helpful for validation. The simulations discussed below all used a cubic box that at the start of each simulation had a edge length of $30 \AA$ and contained 882 water molecules. To avoid any finite time step artifacts, all calibration simulations were conducted with the same time-step as the FEP simulations.

To calculate $\rho$, this box of water was simulated for 30ns in NPT at $300 \mathrm{~K}$ and 1 bar in 5 independent repeats. From these simulations, the number density of SPC water, i.e. the $\rho$ parameter, was calculated to be 0.03248 $\AA^{-3}$. An initial estimate of the hydration free energy of water was obtained by alchemically decoupling a single SPC water molecule from the box of SPC water using 24 lambda windows each run for 40 ns of NPT at 300 $\mathrm{K}$ and 1 bar. Averaging over the last $5 \mathrm{~ns}$ of five repeats produced at estimate of $-6.16 \pm 0.007 \mathrm{kcal} / \mathrm{mol}$. In these calculations, the decoupled water molecule could interact electrostatically with its periodic image. As a water molecule that is deleted with GCMC cannot do so, it was expected that $\mu_{\mathrm{ex}}$ when calculated with GCMC would be slightly more positive than $-6.16 \mathrm{kcal} / \mathrm{mol}$. 


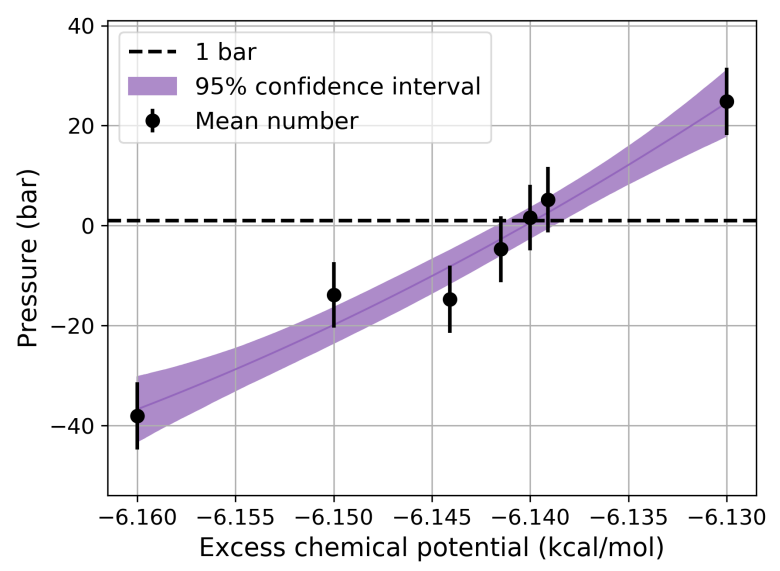

Figure 1: The dependence of the mean pressure of bulk water on the applied chemical potential. Each GCMC simulation of SPC water fixed the value of $\rho$ in equation 35 at $0.03248 \AA^{-3}$ but had a different value of $\mu_{\text {ex }}$. The black dots show the mean pressure with $95 \%$ confidence intervals. The purple line shows the fitted relationship between the applied $\mu_{\mathrm{ex}}$ and the mean pressure that was estimated using a polynomial of degree 2 . The light purple region is the $95 \%$ confidence region of the fit after resampling the mean pressures with bootstrap sampling. The horizontal black dashed line indicates 1 bar. The desired $\mu_{\mathrm{ex}}$ was estimated by finding the value that produced this target pressure.
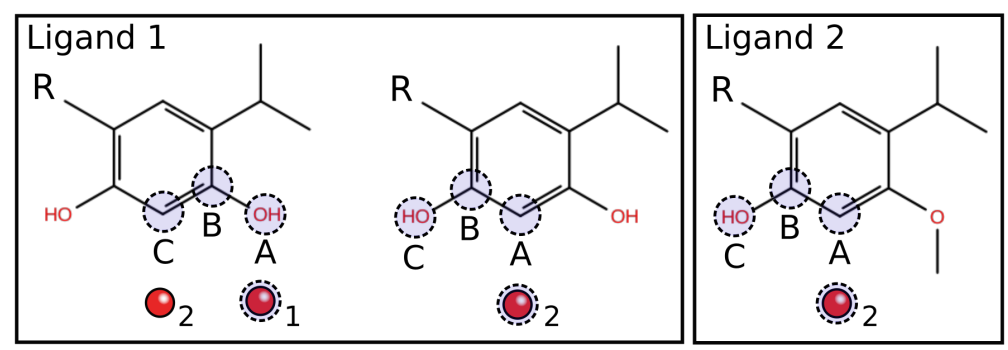

Figure 2: A schematic representation of the relative position restraints used during the alchemical decoupling of water from HSP90. The hydration sites are labelled as 1 and 2. Denoting the water oxygen atom as O, the restraints comprise of a harmonic distance restraint $\mathrm{OA}$, a harmonic angle restraint OAB, and a dihedral angle restraint $\mathrm{OABC}$ for each water molecule. For ligand 1, the restraints on both water molecules were applied at the same time and they were decoupled in numerical order.

The value of $\mu_{\mathrm{ex}}$ was refined by finding the value of the applied chemical potential that had average pressure of 1 bar. Five 30 ns GCMC simulations were performed on the box of water. Each simulation applied a different $\mu$ by varying $\mu_{\mathrm{ex}}$ but fixing the value of $\rho$ at $0.03248 \AA^{-3}$. The relationship between the applied $\mu_{\mathrm{ex}}$ and the pressure was estimated using quadratic interpolation and confidence intervals were estimated using bootstrap sampling. From these calculations, $\mu_{\mathrm{ex}}$ was predicted to be -6.140 [-6.141 -6.139$] \mathrm{kcal} / \mathrm{mol}$, where the upper and lower $95 \%$ confidence intervals are shown within the square brackets. The results of the simulations and fits are shown in Figure 1.

\section{Decoupling water from HSP90}

As described in the main text, water molecules were alchemically decoupled from HSP90 in order to compare relative ligand binding free energies that were calculated in the $\mu \mathrm{VT}$ and NPT ensembles. The results, summarized in Figure 3 of the main text. shows a very close correspondence between the two ensembles. This section contains additional information on the methods used to decouple the water molecules.

While being decoupled, the water molecules were restrained relative to the ligand. For each water molecule, the set of restraints comprised one bond, one angle, and one dihedral angle. Figure 2 shows which ligand atoms were used in the restraints, and these are labelled as A, B, and C. In the following, a water oxygen atom is referred to as $\mathrm{O}$. These relative position restraints on the water molecules were applied during the same alchemical process used to decouple them from the system. When fully decoupled, the free energy to remove the restraints can be 
calculated using the rigid rotor approximation. Let $r$ be the equilibrium bond distance between $\mathrm{O}$ and $\mathrm{A} ; \theta$ be the equilibrium angle between $\mathrm{O}, \mathrm{A}$, and, B; and $\phi$ be the dihedral angle formed by atoms $\mathrm{O}, \mathrm{A}, \mathrm{B}$, and C; and $K_{r}, K_{\theta}$, and $K_{\phi}$ be the respective harmonic force constants. Then, the effective volume of the water when fully decoupled can be shown to be

$$
V_{\text {rest }}=r^{2} \sin (\theta) \frac{\left(2 \pi k_{B} T\right)^{3 / 2}}{\left(K_{r} K_{\theta} K_{\phi}\right)^{1 / 2}},
$$

using the rigid rotor approximation. All the restraint parameters used in the calculations are shown in Table 1.

Denoting the free energy to simultaneously couple and restrain a water molecule as $\Delta G_{\text {calc }}$, the standard state double coupling free energy for a site is was calculated as

$$
\Delta G_{\mathrm{DD}}^{\mathrm{bind}}=\Delta G_{\mathrm{calc}}-\mu_{\mathrm{ex}}-k_{B} T \ln \left(\rho_{\mathrm{bulk}} V_{\text {rest }}\right)
$$

for a single water molecule. As before, $\mu_{\mathrm{ex}}$ denotes the hydration free energy of water and $\rho_{\text {bulk }}$ bulk denotes the density of bulk water. Both $\mu_{\mathrm{ex}}$ and $\rho_{\text {bulk }}$ were calculated during the calibration of the chemical potential.

\begin{tabular}{|c|c|c|c|c|c|c|c|c|}
\hline & $\begin{array}{l}r \\
\AA\end{array}$ & $\begin{array}{c}\theta \\
\operatorname{rad}\end{array}$ & $\begin{array}{c}\phi \\
\mathrm{rad}\end{array}$ & $\begin{array}{c}K_{r} \\
\mathrm{kcal} / \mathrm{mol} / \AA^{2}\end{array}$ & $\begin{array}{c}K_{\theta} \\
\mathrm{kcal} / \mathrm{mol} / \mathrm{rad}^{2}\end{array}$ & $\begin{array}{c}K_{\phi} \\
\mathrm{kcal} / \mathrm{mol} / \mathrm{rad}^{2}\end{array}$ & $\begin{array}{l}\Delta G_{\mathrm{DD}}^{\mathrm{bind}} \\
\mathrm{kcal} / \mathrm{mol}\end{array}$ & $\begin{array}{c}\Delta \Delta G_{\text {solv }} \\
\mathrm{kcal} / \mathrm{mol}\end{array}$ \\
\hline Ligand 1, site 1 & 2.85 & 2.03 & 0.27 & 5.0 & 20.0 & 10.0 & $-6.04 \pm 0.03$ & \multirow{2}{*}{$-7.64 \pm 0.06$} \\
\hline Ligand 1 , site 2 & 3.62 & 1.96 & 0.83 & 5.0 & 20.0 & 10.0 & $-1.60 \pm 0.05$ & \\
\hline Ligand 2, site 2 & 3.68 & 1.98 & 0.86 & 1.0 & 4.0 & 2.0 & $-1.73 \pm 0.03$ & $-1.76 \pm 0.03$ \\
\hline
\end{tabular}
The calculated values of $\Delta G_{\mathrm{DD}}^{\text {bind }}$ averaged over 3 repeats are shown in Table 1.

Table 1: The harmonic restraints applied to the water molecules in the presence of ligands 1 and 2 in HSP90 as well as their double decoupling free energies. The equilibrium bond distance $(r)$, angle $(\theta)$, and improper dihedral $(\phi)$ restraints are shown along with their respective force constants. The average and standard error over 3 repeats are shown for $\Delta G_{\mathrm{DD}}^{\text {bind }}$ and $\Delta \Delta G_{\text {solv }}$. For Ligand 1, equation 34 is used to calculate $\Delta \Delta G_{\text {solv }}$ for the two water molecules, whereas equation 30 (equation 3 in the main text) was used for water molecule bound with Ligand 2.

\section{The accuracy of GCMC-FEP on water disrupting transformations}

\subsection{Expanding the water displacement data set}

\subsubsection{HSP90}

HSP90 has four highly conserved water molecules that are buried deep within the binding site in the apo state. Three of these water molecules, (those that are adjacent to ASN51, SER52, and ASP93), are displaced or disrupted by the two congeneric series considered in this study.

The first congeneric series for HSP90 was taken from the study by Kung et al. in which the growth of a fused ring and the addition of substituents gradually displace the 3 waters [13]. The experimental binding affinities were taken from a high-throughput competition assay. These measurements should be interpreted with some caution; the binding free energies were measured with ITC on four compounds and these values had an RMSD of $0.86 \mathrm{kcal} / \mathrm{mol}$ compared to the faster technique. The protein structure was taken from PDB entry 3RLP (which contains the 3 buried water molecules). The ligand binding modes were based on the binding poses found in PDB entries 3RLP, 3RLQ, and 3RLR.

In the second congeneric series for HSP90, four ligands were taken from Woodhead et al. [19], two of these ligands are the same as those used for the comparison of GCMC with alchemical decoupling (see Figure 3 of the main text). All ligands in this series displace the buried water that is closest to ASN51, and the ligands used in this study have different interactions with the two remaining waters molecules, with one ligand sterically displacing one of them. The ligands had their affinities measured with ITC. The binding poses of the ligands was taken from PDB entries 2XAB, 2XJG, and 2XJJ; the protein structure was taken from 2XJJ.

\subsubsection{Chk1}

Ligands were taken from Fraley et al. in which different rings and ring substitutions interact with a buried pocket that contains three water molecules [6]. While some ligands do not sterically displace the waters, some or all of 
the waters can be displaced by the modifications in this series. The ligands were aligned to the crystallographic ligand in the structure that accompanied the publication (PDB entry 2HOG). In that structure, one of the buried water molecules is displaced. Despite knowing the overall binding modes of the ligands, the orientations of the ligand perturbations required further modelling. The orientation of one of the ligand modifications (compound 24) was taken from PDB entry 2C3K with the rest predicted using Glide [7].

Some of the ligands contained aromatic nitrogens whose tautomeric state was uncertain, for instance, ligand 21 in Figure 4 of the main text. Tautomers for all ligands were generated with Epik [8]. After using Epik, uncertainty remained for ligands 13 and 21 which was resolved with Schrödinger's macro-pKa prediction protocol [16].

As 2 HOG is missing residues 44 to 50 as well as the side chain for TYR 20, PDB entry 2E9V (resolved at $1.9 \AA$, $0.65 \AA$ backbone RMSD from $2 \mathrm{HOG}$ ) was used as the starting protein structure for FEP. The oxygen positions of the unperturbed three-water network were also taken from $2 \mathrm{E} 9 \mathrm{~V}$.

\subsubsection{Urokinase}

Four ligands were taken Katz et al. [10,11] to explore the displacement of the buried water (in the vicinity of ASP 189) by F and Cl. Although more chemical substituents were synthesized and assayed by Katz et al., the binding affinities of the four ligands selected for this study were measured more rigorously than many others.

The protein structures and ligand binding modes were taken from PDB entries 1GJ7 and 1GJB. PDB entries $1 \mathrm{GJ} 7$ and 1GJB show that the addition of $\mathrm{Cl}$ to the scaffold shifts the binding mode of the ligand towards ASP 189 and the buried hydration site water by approximately $1 \AA$. As a result, the ligand pose in 1 GJB was used as the basis for the FEP map in the presence of the buried water molecule, and pose in IGJ7 was used for the FEP map in the absence of the water molecule. ASN 192 was added to the REST region as both 1G7J and $1 \mathrm{G} 7 \mathrm{~B}$ resolve this residue in 2 conformations. Experimental evidence accumulated by Katz et al. indicates that HIS 57 and the phenol in the ligand scaffold form a salt bridge at pH 7 . Thus, HIS 57 was prepared in the biprotonated state and the phenol oxygen was deprotonated.

\subsubsection{Thrombin}

Baum et al. explored 26 small modifications to a single scaffold where all except one all consist of small additions to a phenyl ring [2]. A buried water molecule (adjacent to ASP 189) that is present with the unadorned phenyl group (PDB entry 2ZFF) is displaced by the addition of $\mathrm{CH}$, F, or $\mathrm{Cl}$ in the meta position (PDB entries 2ZF0, 2ZDZ, and 2ZC9 respectively). The binding affinities of all the ligands were measured using a kinetic competition assay and a subset of 12 ligands also had their affinities measured with ITC. The absolute difference of the measured affinities between the two techniques range from $0.02 \mathrm{kcal} / \mathrm{mol}$ to $0.80 \mathrm{kcal} / \mathrm{mol}$ with an RMSD of $0.50 \mathrm{kcal} / \mathrm{mol}$. The 12 ligands that were assayed with ITC were used previously by a previous FEP+ study [18]. The experimental affinities were taken from the competition assay given its greater coverage of ligands. Only the neutral ligands that had unambiguous stereochemistry (a total of 24) were used in this work.

PDB entry 2ZFF was used for the protein structure and as the basis for the binding modes of the ligands. Restraints were placed on a $\mathrm{Na}+$ ion adjacent to ASP 189 to prevent its diffusion. As indicated by Figure 4 in the main text, ligand 5 was placed at the center of the map with all ligands connected to it. The orientation of most of the ligands with substitutes in the ortho and meta position was unknown and highly unlikely to change during the course of the simulation. Two orientations were added to the FEP map, both with an edge to ligand 5 and edge between them. The relative free energies between these modes were combined into a single relative free energy prediction using the same scheme as established previously [12,15].

\subsubsection{Scytalone dehydratase}

The ligands developed by Chen et al. [3] for scytalone dehydratase are commonly used for validating protocols that displace water molecules (see, for instance Michel et al. [14,17]). This series explores nitrogen substitutions to quinoline derivatives as well as the growth of a cyano-nitrile group into a conserved hydration site. PDB entry 3STD - in which the buried water has been displaced - was used as the protein model for FEP and as the basis for binding modes of the ligands. The location of the water oxygen atom was taken from PDB entry 5STD.

Based on an experimental measurement of a close chemical analogue [9], the quinoline nitrogen in ligand 3d of this set (shown in Figure 4 of the main text) is expected to have a pKa of 8.0. As the experimental assay was conducted at pH 7 [3], this ligand will be protonated in solvent. A preliminary FEP + calculation between 
the protonated and neutral form of ligand 3d when bound to the protein predicted that the pKa lowers by roughly 9 units. Based on this result, it was assumed that the remaining ligands bind in their neutral forms. Schrödinger's method to determine macro-pKas was run on the remaining ligands to estimate the pKas of the aromatic nitrogens [16]. These predictions, shown in Table 4 of the main text, have an expected error of about 1 pKa unit, making the protonation state of ligands $2 \mathrm{~d}, 6 \mathrm{~d}$, and $8 \mathrm{~d}$ in solvent uncertain. A correction, described previously [5], was applied to predicted relative free energies to account for the pKa of the ligands in solvent. The uncertainty in the macro-pKa predictions - particularly for $2 \mathrm{~d}, 6 \mathrm{~d}$, and $8 \mathrm{~d}$ - adds a degree of uncertainty to the scytalone dehydratase FEP predictions that is not present in the other data sets.

\subsubsection{Bromodomains Brd4(1) and Taf1(2)}

There are four hydration sites that are present in all apo bromodomains whose propensity for displacement has been previously investigated by GCMC [1]. Eight ligands were taken from a study by Crawford et al. in which an alkyl chain was grown into the buried water pocket for a large number of bromodomains [4]. Crystallographic structures that accompanied the work show some of the four waters are displaced for Brd4(1) and Taf1(2). The same FEP ligand map was used for both $\operatorname{Brd4}(1)$ and $\operatorname{Taf1}(2)$.

For Brd4(1) and Taf1(2), PDB entries 5I80 and 5I29 were used as the structure that contained all four buried molecules, respectively, whereas PDB 5I88 and 5I1Q were used as the structure whose water molecules had been displaced and disrupted. The ligand binding modes were based on 5I88 and 5I1Q for Brd4(1) and Taf1(2), respectively.

\subsection{Supplemental Results}

\begin{tabular}{l|c|ccc|ccc|}
\multirow{2}{*}{ Edge } & Exp. & \multicolumn{3}{|c}{ Without overlapping water } & \multicolumn{3}{c}{ With overlapping water } \\
& & $\mu \mathrm{VT}$ & $\mathrm{NPT}$ & NPT pre-solvate & $\mu \mathrm{VT}$ & $\mathrm{NPT}$ & NPT pre-solvate \\
\hline i & -0.20 & $2.52 \pm 0.06$ & $1.14 \pm 0.05$ & $3.49 \pm 0.09$ & $2.20 \pm 0.07$ & $5.40 \pm 0.14$ & $1.97 \pm 0.13$ \\
ii & -2.23 & $-4.00 \pm 0.03$ & $3.45 \pm 0.05$ & $4.09 \pm 0.10$ & $-3.94 \pm 0.03$ & $3.36 \pm 0.07$ & $4.02 \pm 0.06$ \\
iii & -1.42 & $-1.47 \pm 0.16$ & $-1.65 \pm 0.34$ & $-2.22 \pm 0.19$ & $-1.90 \pm 0.16$ & $-0.34 \pm 0.22$ & $-1.28 \pm 0.11$ \\
iv & 1.23 & $1.83 \pm 0.06$ & $1.04 \pm 0.05$ & $1.34 \pm 0.10$ & $1.57 \pm 0.06$ & $1.03 \pm 0.05$ & $1.19 \pm 0.10$ \\
v & -1.98 & $-2.55 \pm 0.14$ & $3.87 \pm 0.07$ & $-3.71 \pm 0.12$ & $-2.42 \pm 0.16$ & $-3.93 \pm 0.08$ & $-2.42 \pm 0.10$ \\
vi & 3.79 & $4.50 \pm 0.09$ & $3.60 \pm 0.06$ & $3.66 \pm 0.06$ & $4.47 \pm 0.10$ & $3.86 \pm 0.06$ & $3.64 \pm 0.08$ \\
vii & 0.61 & $-1.69 \pm 0.10$ & $-2.47 \pm 0.25$ & $-1.64 \pm 0.16$ & $-2.35 \pm 0.08$ & $-1.04 \pm 0.14$ & $-1.99 \pm 0.25$ \\
viii & -1.43 & $-0.25 \pm 0.08$ & $-0.73 \pm 0.07$ & $-0.38 \pm 0.07$ & $-0.60 \pm 0.10$ & $-0.72 \pm 0.12$ & $-0.86 \pm 0.10$ \\
ix & -2.00 & $0.37 \pm 0.17$ & $-0.84 \pm 0.15$ & $-1.18 \pm 0.10$ & $1.04 \pm 0.34$ & $2.11 \pm 0.27$ & $1.20 \pm 0.24$ \\
x & 1.92 & $4.21 \pm 0.24$ & $4.78 \pm 0.17$ & $3.92 \pm 0.21$ & $3.68 \pm 0.25$ & $4.53 \pm 0.39$ & $4.58 \pm 0.26$
\end{tabular}

Table 2: The experimental (Exp.) and predicted relative free energies for the 10 transformations in the Wahl and Smieško data set. The naming scheme for each transformation (Edge) follows is the same as the original study. All values are in $\mathrm{kcal} / \mathrm{mol}$.

\begin{tabular}{l|c|ccc} 
System & Number of edges & $\mu \mathrm{VT}$ & NPT & NPT pre-solvate \\
\hline HSP90 (Woodhead et al.) & 5 & $0.28 \pm 0.05$ & $2.44 \pm 0.71$ & $0.78 \pm 0.14$ \\
HSP90 (Kung et al.) & 18 & $1.96 \pm 0.27$ & $4.21 \pm 1.23$ & $1.84 \pm 0.30$ \\
Urokinase & 6 & $0.71 \pm 0.13$ & $0.68 \pm 0.10$ & $1.02 \pm 0.22$ \\
Thrombin* & 21 & $1.02 \pm 0.17$ & $1.24 \pm 0.32$ & $1.10 \pm 0.20$ \\
Taf1(2) & 11 & $0.79 \pm 0.11$ & $0.67 \pm 0.09$ & $0.65 \pm 0.08$ \\
Brd4(1) & 11 & $2.31 \pm 0.29$ & $2.23 \pm 0.27$ & $2.19 \pm 0.27$ \\
Scytalone dehydratase & 12 & $1.74 \pm 0.19$ & $1.65 \pm 0.29$ & $1.74 \pm 0.23$ \\
Chk1 & 19 & $0.76 \pm 0.10$ & $1.05 \pm 0.16$ & $0.91 \pm 0.14$
\end{tabular}

Table 3: The RMSE (in $\mathrm{kcal} / \mathrm{mol}$ ) on each buried water perturbing data set when the starting structure does not contain water molecules that overlap with the ligands. The 'number of edges' column refers to the number of perturbations in each FEP map. * For thrombin, the number of edges and RMSEs are shown after FEP predictions on different binding modes of the same ligands have been aggregated into a single value. 


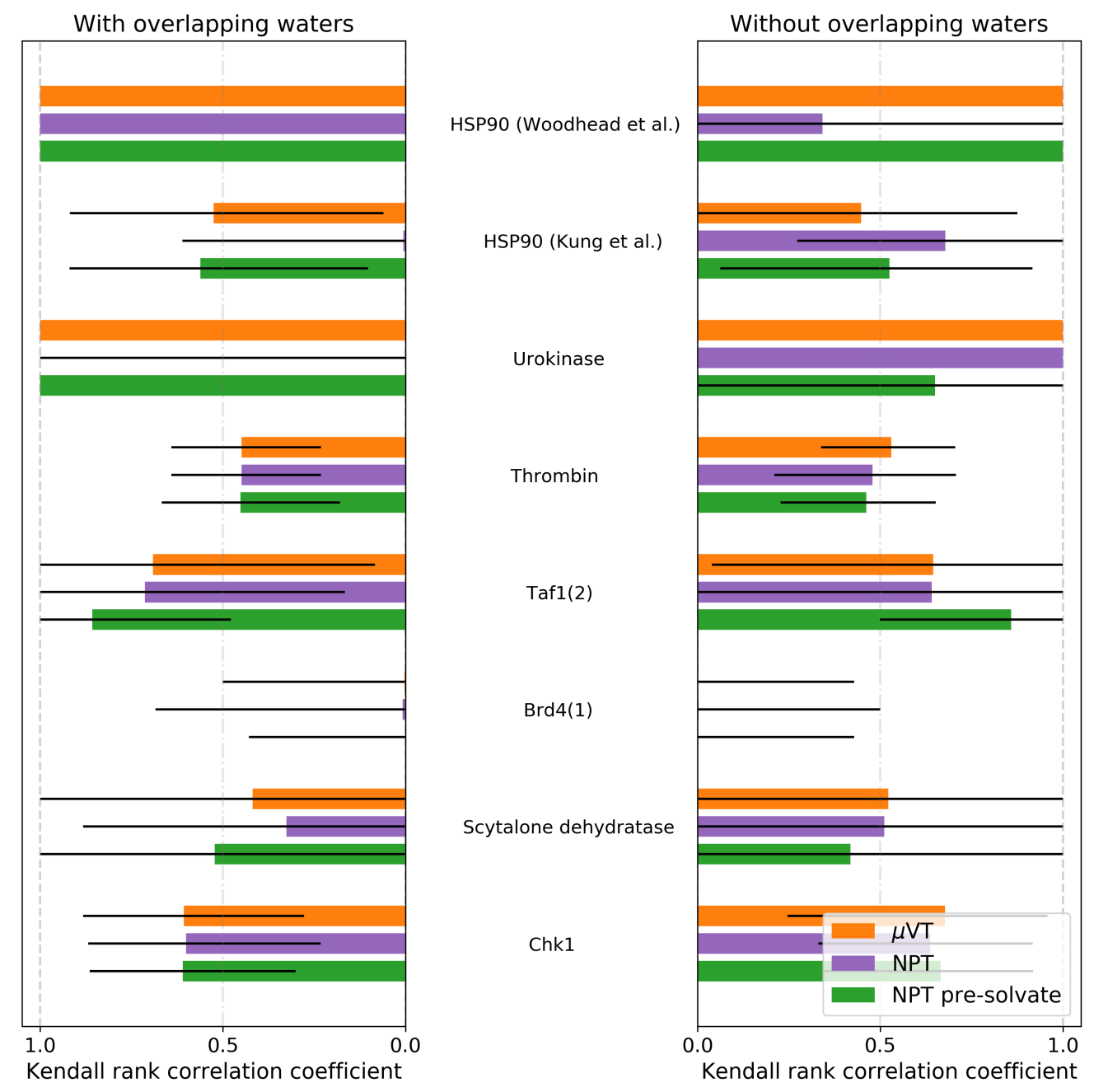

Figure 3: The Kendall tau rank correlation for each system in the water disruption data sets. 

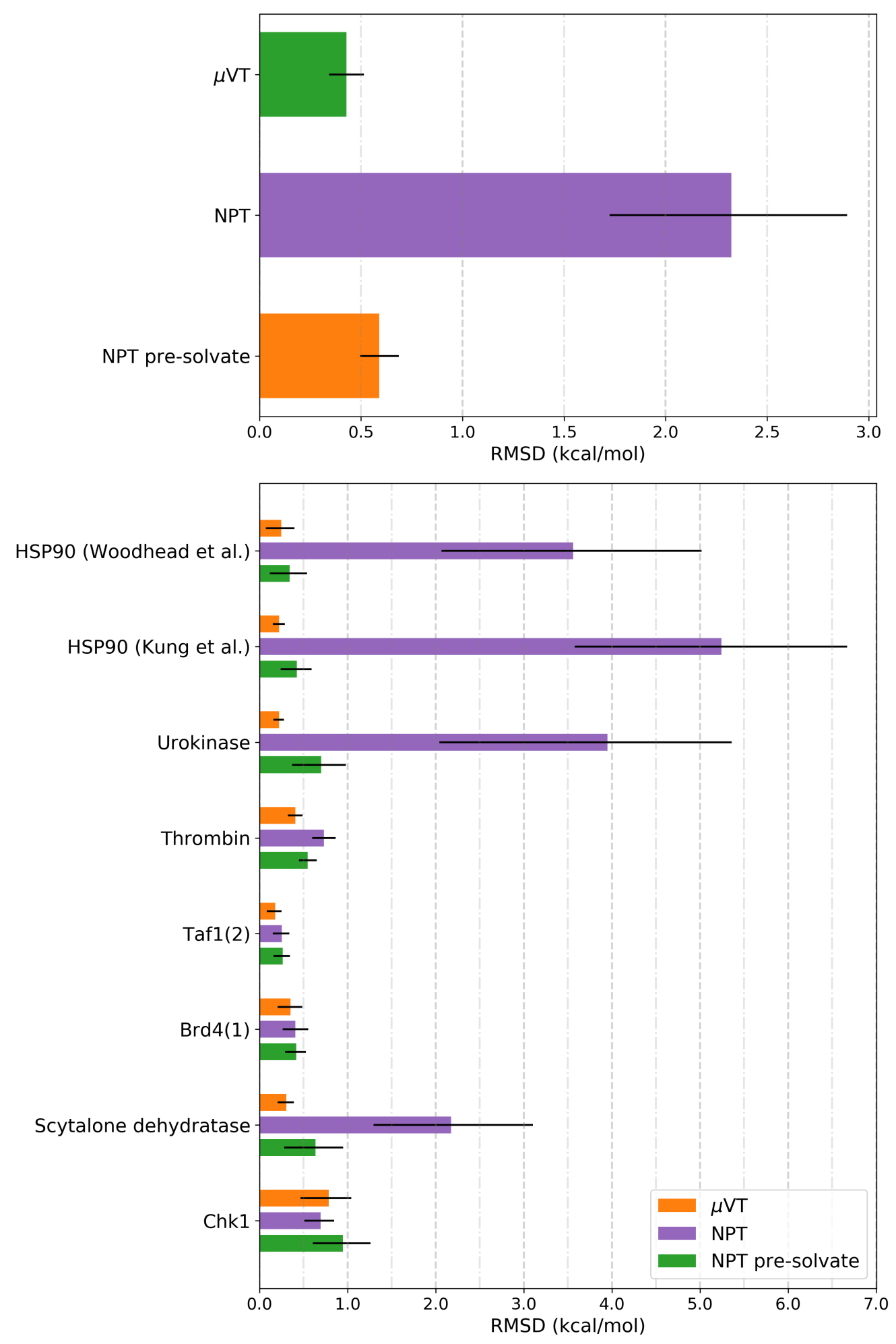

Figure 4: The root-mean-squared-deviations (RMSDs) between free energy perturbations that start with and without buried water molecules for the three protocols considered. Top panel: the RMSDs that have been evaluated using all edges. Bottom panel: the RMSDs that have been evaluated for each system.

\section{References}

[1] Matteo Aldeghi, Gregory A. Ross, Michael J. Bodkin, Jonathan W. Essex, Stefan Knapp, and Philip C. Biggin. Large-scale analysis of water stability in bromodomain binding pockets with grand canonical Monte Carlo. Commun. Chem., 2018, 1, 19. 
[2] Bernhard Baum, Menshawy Mohamed, Mohamed Zayed, Christof Gerlach, Andreas Heine, David Hangauer, and Gerhard Klebe. More than a Simple Lipophilic Contact: A Detailed Thermodynamic Analysis of Nonbasic Residues in the S1 Pocket of Thrombin. J. Mol. Biol., 2009, 390, 56-69.

[3] James M Chen, Simon L Xu, Zdzislaw Wawrzak, Gregory S Basarab, and Douglas B Jordan. StructureBased Design of Potent Inhibitors of Scytalone Dehydratase: Displacement of a Water Molecule from the Active Site. Biochemistry, 1998, 37, 17735-17744.

[4] Terry D. Crawford, Vickie Tsui, E. Megan Flynn, Shumei Wang, Alexander M. Taylor, Alexandre Côté, James E. Audia, Maureen H. Beresini, Daniel J. Burdick, Richard Cummings, Les A. Dakin, Martin Duplessis, Andrew C. Good, Michael C. Hewitt, Hon Ren Huang, Hariharan Jayaram, James R. Kiefer, Ying Jiang, Jeremy Murray, Christopher G. Nasveschuk, Eneida Pardo, Florence Poy, F. Anthony Romero, Yong Tang, Jian Wang, Zhaowu Xu, Laura E. Zawadzke, Xiaoyu Zhu, Brian K. Albrecht, Steven R. Magnuson, Steve Bellon, and Andrea G. Cochran. Diving into the Water: Inducible Binding Conformations for BRD4, TAF1(2), BRD9, and CECR2 Bromodomains. J. Med. Chem., 2016, 59, 5391-5402.

[5] César De Oliveira, Haoyu S. Yu, Wei Chen, Robert Abel, and Lingle Wang. Rigorous Free Energy Perturbation Approach to Estimating Relative Binding Affinities between Ligands with Multiple Protonation and Tautomeric States. J. Chem. Theory Comput., 2019, 15, 424-435.

[6] Mark E. Fraley, Justin T. Steen, Edward J. Brnardic, Kenneth L. Arrington, Keith L. Spencer, Barbara A. Hanney, Yuntae Kim, George D. Hartman, Steven M. Stirdivant, Bob A. Drakas, Keith Rickert, Eileen S. Walsh, Kelly Hamilton, Carolyn A. Buser, James Hardwick, Weikang Tao, Stephen C. Beck, Xianzhi Mao, Robert B. Lobell, Laura Sepp-Lorenzino, Youwei Yan, Mari Ikuta, Sanjeev K. Munshi, Lawrence C. Kuo, and Constantine Kreatsoulas. 3-(Indol-2-yl)indazoles as Chek1 kinase inhibitors: Optimization of potency and selectivity via substitution at C6. Bioorganic Med. Chem. Lett., 2006, 16, 6049-6053.

[7] Richard A. Friesner, Robert B. Murphy, Matthew P. Repasky, Leah L. Frye, Jeremy R. Greenwood, Thomas A. Halgren, Paul C. Sanschagrin, and Daniel T. Mainz. Extra Precision Glide: Docking and Scoring Incorporating a Model of Hydrophobic Enclosure for Protein-Ligand Complexes. J. Med. Chem., 2006, 49, 6177-6196.

[8] Jeremy R. Greenwood, David Calkins, Arron P. Sullivan, and John C. Shelley. Towards the comprehensive, rapid, and accurate prediction of the favorable tautomeric states of drug-like molecules in aqueous solution. J. Comput. Aided. Mol. Des., 2010, 24, 591-604.

[9] Catherine H. Kaschula, Timothy J. Egan, Roger Hunter, Nicoletta Basilico, Silvia Parapini, Donatella Taramelli, Erica Pasini, and Diego Monti. Structure - Activity relationships in 4-aminoquinoline antiplasmodials. The role of the group at the 7-position. J. Med. Chem., 2002, 45, 3531-3539.

[10] Bradley A. Katz, Kyle Elrod, Erik Verner, Richard L. Mackman, Christine Luong, William D. Shrader, Martin Sendzik, Jeffrey R. Spencer, Paul A. Sprengeler, Aleks Kolesnikov, Vincent W.F. Tai, Hon C. Hui, J. Guy Breitenbucher, Darin Allen, and James W. Janc. Elaborate manifold of short hydrogen bond arrays mediating binding of active site-directed serine protease inhibitors. J. Mol. Biol., 2003, 329, 93-120.

[11] Bradley A. Katz, Paul A. Sprengeler, Christine Luong, Erik Verner, Kyle Elrod, Matt Kirtley, James Janc, Jeffrey R. Spencer, J. Guy Breitenbucher, Hon Hui, Danny McGee, Darin Allen, Arnold Martelli, and Richard L. Mackman. Engineering inhibitors highly selective for the S1 sites of Ser190 trypsin-like serine protease drug targets. Chem. Biol., 2001, 8, 1107-1121.

[12] Joseph W. Kaus, Edward Harder, Teng Lin, Robert Abel, J. Andrew McCammon, and Lingle Wang. How to deal with multiple binding poses in alchemical relative protein-ligand binding free energy calculations. J. Chem. Theory Comput., 2015, 11, 2670-2679.

[13] Pei Pei Kung, Piet Jan Sinnema, Paul Richardson, Michael J. Hickey, Ketan S. Gajiwala, Fen Wang, Buwen Huang, Guy McClellan, Jeff Wang, Karen Maegley, Simon Bergqvist, Pramod P. Mehta, and Robert Kania. Design strategies to target crystallographic waters applied to the Hsp90 molecular chaperone. Bioorganic Med. Chem. Lett., 2011, 21, 3557-3562.

[14] Julien Michel, Julian Tirado-Rives, and William L. Jorgensen. Energetics of displacing water molecules from protein binding sites: consequences for ligand optimization. J. Am. Chem. Soc., 2009, 131, 1540315411.

[15] David L Mobley, John D Chodera, and Ken A Dill. On the use of orientational restraints and symmetry corrections in alchemical free energy calculations. J. Chem. Phys, 2006, 125, 084902.

[16] Dean M. Philipp, Mark A. Watson, Haoyu S. Yu, Thomas B. Steinbrecher, and Art D. Bochevarov. Quantum chemical pK a prediction for complex organic molecules. Int. J. Quantum Chem., 2018, 118, 1-8. 
[17] Gregory A Ross, Michael S Bodnarchuk, and Jonathan W Essex. Water Sites, Networks, And Free Energies with Grand Canonical Monte Carlo. J. Am. Chem. Soc., 2015, 137, 14930-14943.

[18] Lingle Wang, Yujie Wu, Yuqing Deng, Byungchan Kim, Levi Pierce, Goran Krilov, Dmitry Lupyan, Shaughnessy Robinson, Markus K Dahlgren, Jeremy Greenwood, Donna L Romero, Craig Masse, Jennifer L Knight, Thomas Steinbrecher, Thijs Beuming, Wolfgang Damm, Ed Harder, Woody Sherman, Mark Brewer, Ron Wester, Mark Murcko, Leah Frye, Ramy Farid, Teng Lin, David L Mobley, William L Jorgensen, Bruce J Berne, Richard A Friesner, and Robert Abel. Accurate and Reliable Prediction of Relative Ligand Binding Potency in Prospective Drug Discovery by Way of a Modern Free-Energy Calculation Protocol and Force Field. J. Am. Chem. Soc., 2015, 13\%, 2695-27035.

[19] Andrew J. Woodhead, Hayley Angove, Maria G. Carr, Gianni Chessari, Miles Congreve, Joseph E. Coyle, Jose Cosme, Brent Graham, Philip J. Day, Robert Downham, Lynsey Fazal, Ruth Feltell, Eva Figueroa, Martyn Frederickson, Jonathan Lewis, Rachel McMenamin, Christopher W. Murray, M. Alistair O’Brien, Lina Parra, Sahil Patel, Theresa Phillips, David C. Rees, Sharna Rich, Donna Michelle Smith, Gary Trewartha, Mladen Vinkovic, Brian Williams, and Alison J.A. Woolford. Discovery of (2,4-Dihydroxy-5-isopropylphenyl)-[5-(4-methylpiperazin-1- ylmethyl)-1,3-dihydroisoindol-2-yl]methanone (AT13387), a novel inhibitor of the molecular chaperone Hsp90 by fragment based drug design. J. Med. Chem., 2010, 53, 5956-5969. 